\title{
Correction to: Metformin and sulodexide restore cardiac microvascular perfusion capacity in diet-induced obese rats
}

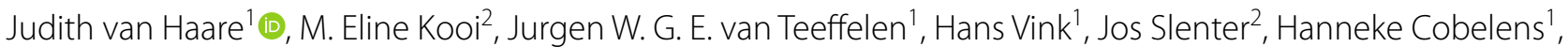
Gustav J. Strijkers ${ }^{4}$, Dennis Koehn ${ }^{5}$, Mark J. Post ${ }^{1}$ and Marc van Bilsen ${ }^{1,3^{*}}$

\section{Correction to: Cardiovasc Diabetol (2017) 16:47}

https://doi.org/10.1186/s12933-017-0525-7

Following publication of the original article [1], the authors regret errors in Figs. $2 b-d$. In these figures the images of the representative Akt and phospho-Akt (pAkt) signals should be replaced with the appropriate images. The representative images shown here are correct. The changes do not affect the scientific conclusion and significance of the article. 
b

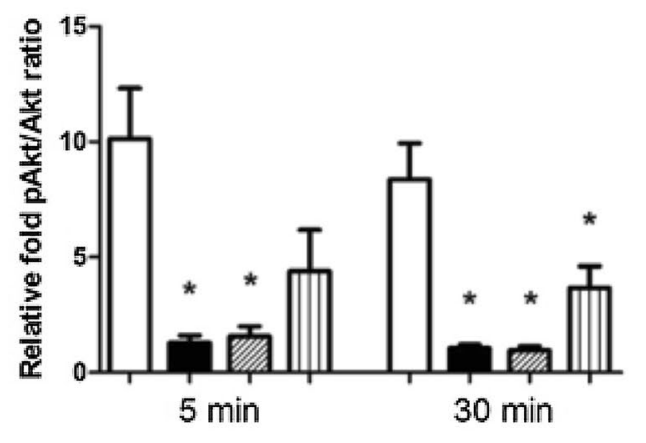

C

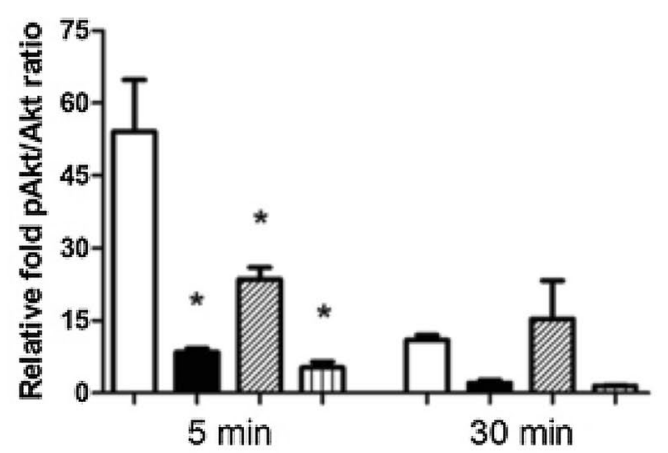

d

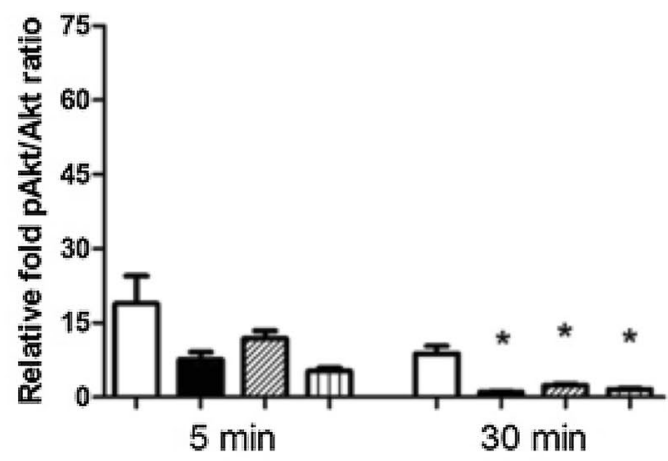

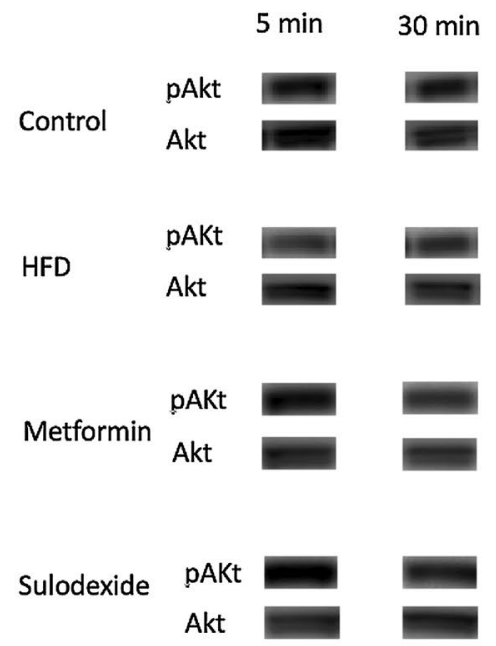

$5 \min \quad 30 \min$

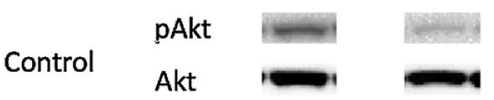

$\square$ Control

HFD

metformin

m Sulodexide

HFD

pAKt

Akt

$\infty$

-

$\longrightarrow$

DAKt

Metformin Akt

Sulodexide

pAKt

Akt

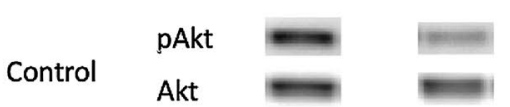

HFD

pAKt

Akt

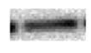

m Sulodexide

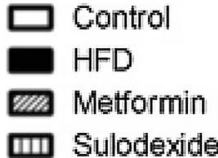

Metformin $\begin{aligned} & \text { pAKt } \\ & \text { Akt }\end{aligned}$

Sulodexide

pAKt

Akt 


\section{Author details}

${ }^{1}$ Department of Physiology, Maastricht University, P.O. Box 616, 6200

MD Maastricht, The Netherlands. ${ }^{2}$ Department of Radiology and Nuclear

Medicine, Maastricht University, P.O. Box 616, 6200 MD Maastricht, The Netherlands. ${ }^{3}$ Department of Cardiology, CARIM School for Cardiovascular Diseases,

Maastricht University, P.O. Box 616, 6200 MD Maastricht, The Netherlands. ${ }^{4}$ Biomedical Engineering and Physics, Academic Medical Center, P.O. Box 22700 ,

1100 DE Amsterdam, The Netherlands. ${ }^{5}$ Pie Medical Imaging, P.O. Box 1132 , 6201 BC Maastricht, The Netherlands.

Received: 6 February 2021 Accepted: 6 February 2021

Published online: 26 February 2021

\section{Reference}

1. van Haare J, Kooi ME, van Teeffelen JWGE, Vink H, Slenter J, Cobelens H, Strijkers GJ, Koehn D, Post MJ, van Bilsen M. Metformin and sulodexide restore cardiac microvascular perfusion capacity in diet-induced obese rats. Cardiovasc Diabetol. 2017;16:47. https://doi.org/10.1186/s1293 3-017-0525-7.

\section{Publisher's Note}

Springer Nature remains neutral with regard to jurisdictional claims in published maps and institutional affiliations. 